\title{
MAPPING THE RELEASE OF VOLATILES IN THE INNER COMAE OF COMETS C/2012 F6 (LEMMON) AND C/2012 S1 (ISON) USING THE ATACAMA LARGE MILLIMETER/SUBMILLIMETER ARRAY
}

\author{
M. A. Cordiner ${ }^{1,2}$, A. J. Remijan ${ }^{3}$, J. Boissier ${ }^{4}$, S. N. Milam ${ }^{1}$, M. J. Mumma ${ }^{1}$, S. B. Charnley ${ }^{1}$, L. Paganini ${ }^{1,2}$, \\ G. Villanueva ${ }^{1,2}$, D. Bockelée-Morvan ${ }^{5}$, Y.-J. Kuan ${ }^{6,7}$, Y.-L. Chuang ${ }^{6}$, D. C. Lis ${ }^{8,9}$, N. Biver ${ }^{5}$, \\ J. CROvisieR $^{5}$, D. Minniti ${ }^{10,11}$, AND I. M. Coulson ${ }^{12}$ \\ ${ }^{1}$ Goddard Center for Astrobiology, NASA Goddard Space Flight Center, 8800 Greenbelt Road, Greenbelt, MD 20771, USA; \\ martin.cordiner@nasa.gov \\ ${ }^{2}$ Department of Physics, Catholic University of America, 620 Michigan Avenue NE, Washington, DC 20064, USA \\ ${ }^{3}$ National Radio Astronomy Observatory, Charlottesville, VA 22903, USA \\ ${ }^{4}$ IRAM, 300 Rue de la Piscine, F-38406 Saint Martin d'Heres, France \\ ${ }^{5}$ LEISA, Observatoire de Paris, CNRS, UPMC, Université Paris-Diderot, 5 Place Jules Janssen, F-92195 Meudon, France \\ ${ }^{6}$ National Taiwan Normal University, Taipei 116, Taiwan, Republic of China \\ ${ }^{7}$ Institute of Astronomy and Astrophysics, Academia Sinica, Taipei 106, Taiwan, Republic of China \\ ${ }^{8}$ Sorbonne Universités, Université Pierre et Marie Curie, Paris 6, CNRS, Observatoire de Paris, UMR 8112, LERMA, F-75014 Paris, France \\ ${ }^{9}$ Cahill Center for Astronomy and Astrophysics 301-17, California Institute of Technology, Pasadena, CA 91125, USA \\ ${ }^{10}$ Pontifica Universidad Catolica de Chile, Santiago, Chile \\ ${ }^{11}$ Departamento de Ciencias Fisicas, Universidad Andres Bello, Republica 220, Santiago, Chile \\ 12 Joint Astronomy Centre, Hilo, HI 96720, USA \\ Received 2014 June 17; accepted 2014 July 9; published 2014 August 11
}

\begin{abstract}
Results are presented from the first cometary observations using the Atacama Large Millimeter/Submillimeter Array (ALMA), including measurements of the spatially resolved distributions of $\mathrm{HCN}, \mathrm{HNC}, \mathrm{H}_{2} \mathrm{CO}$, and dust within the comae of two comets: C/2012 F6 (Lemmon) and C/2012 S1 (ISON), observed at heliocentric distances of $1.5 \mathrm{AU}$ and $0.54 \mathrm{AU}$, respectively. These observations (with angular resolution $\approx 0$ '.5), reveal an unprecedented level of detail in the distributions of these fundamental cometary molecules, and demonstrate the power of ALMA for quantitative measurements of the distributions of molecules and dust in the inner comae of typical bright comets. In both comets, $\mathrm{HCN}$ is found to originate from (or within a few hundred kilometers of) the nucleus, with a spatial distribution largely consistent with spherically symmetric, uniform outflow. By contrast, the HNC distributions are clumpy and asymmetrical, with peaks at cometocentric radii $\sim 500-1000 \mathrm{~km}$, consistent with release of HNC in collimated outflow(s). Compared to $\mathrm{HCN}$, the $\mathrm{H}_{2} \mathrm{CO}$ distribution in comet Lemmon is very extended. The interferometric visibility amplitudes are consistent with coma production of $\mathrm{H}_{2} \mathrm{CO}$ and $\mathrm{HNC}$ from unidentified precursor material(s) in both comets. Adopting a Haser model, the $\mathrm{H}_{2} \mathrm{CO}$ parent scale length is found to be a few thousand kilometers in Lemmon and only a few hundred kilometers in ISON, consistent with the destruction of the precursor by photolysis or thermal degradation at a rate that scales in proportion to the solar radiation flux.
\end{abstract}

Key words: comets: individual (C/2012 S1 (ISON), C/2012 F6 (Lemmon)) - techniques: interferometric

Online-only material: color figures, supplemental data

\section{INTRODUCTION}

Astronomical observations of comets provide an important means to study some of the oldest, most pristine material in our solar system. The bulk of cometary ices are believed to be relatively unprocessed, having accreted at around the time the planets formed (c. $4.5 \mathrm{Gyr}$ ago), and have remained in a frozen, relatively quiescent state since then. Depending on the degree of subsequent thermal and radiative processing, some comets likely contain pristine material from the solar nebula or prior interstellar cloud. Studies of cometary ices thus provide unique information on the physics and chemistry of the early stages of the solar system's evolution. Comets could also have been important for initiating prebiotic chemistry on the early Earth, and their study provides crucial details on the link between interstellar ices and planetary material (Ehrenfreund \& Charnley 2000; Mumma \& Charnley 2011).

Use of gas-phase coma observations as a probe of cometary ice composition requires a complete understanding of the gasrelease mechanisms. However, previous observations have been unable to ascertain the precise origins of key coma species, including hydrogen cyanide $(\mathrm{HCN})$, hydrogen iso-cyanide ( $\mathrm{HNC})$, and formaldehyde $\left(\mathrm{H}_{2} \mathrm{CO}\right)$, and details regarding the possible formation of these species in the coma are not well understood. Hydrogen cyanide is a trace volatile commonly assumed to originate in the nucleus, but a significant source of $\mathrm{HCN}$ in the coma has not yet been ruled out. In comet 103P/ Hartley 2, evidence was found for the release of $\mathrm{HCN}$ from icy grains at nucleocentric distances up to $1000 \mathrm{~km}$ (Boissier et al. 2014). Hydrogen iso-cyanide in cometary comae has been discussed as a possible tracer of pristine interstellar material pre-dating the origin of the solar system (Irvine et al. 1996). However, variations in HNC production rates with heliocentric distance are more consistent with its production in the coma (Biver et al. 1997; Irvine et al. 1998; Lis et al. 2008). Detailed chemical modeling (Rodgers \& Charnley 2001, 2005) has thus far failed to conclusively identify the origin of cometary HNC. Formaldehyde is ubiquitous in dense interstellar clouds and its study in comets is of major interest to astrochemistry and astrobiology. The production of $\mathrm{H}_{2} \mathrm{CO}$ in the coma from a distributed (extended) source has been observed in several comets (Biver et al. 1999; Cottin et al. 2004; Milam et al. 2006), and a 
Table 1

Observation Parameters

\begin{tabular}{|c|c|c|c|c|c|c|c|c|c|c|c|c|}
\hline Comet & Setting & Date & UT Time & $\begin{array}{l}\text { Int. time }{ }^{\mathrm{a}} \\
(\mathrm{min})\end{array}$ & $\begin{array}{c}r_{H}^{\mathrm{b}} \\
(\mathrm{AU})\end{array}$ & $\begin{array}{c}\Delta^{\mathrm{c}} \\
(\mathrm{AU})\end{array}$ & $\begin{array}{l}\phi^{\mathrm{d}} \\
\left(^{\circ}\right)\end{array}$ & $\begin{array}{c}\bar{v}^{\mathrm{e}} \\
(\mathrm{GHz})\end{array}$ & Ants. ${ }^{\mathrm{f}}$ & $\begin{array}{c}\text { Baselines }^{g} \\
\text { (m) }\end{array}$ & $\begin{array}{c}\theta_{\min } \mathrm{h} \\
\left({ }^{\prime \prime}\right)\end{array}$ & $\begin{array}{l}\mathrm{PWV}^{\mathrm{i}} \\
(\mathrm{mm})\end{array}$ \\
\hline C/2012 F6 & 1 & 2013 Jun 1 & $11: 52-12: 32$ & 29.6 & 1.47 & 1.75 & 35 & 346.6 & 30 & $15.2-1284$ & $0.88 \times 0.54$ & 0.83 \\
\hline C/2012 F6 & 2 & 2013 Jun 2 & $12: 11-12: 33$ & 17.1 & 1.48 & 1.75 & 35 & 357.5 & 28 & $21.4-2733$ & $0.78 \times 0.54$ & 0.44 \\
\hline $\mathrm{C} / 2012 \mathrm{~S} 1$ & 1 & 2013 Nov 17 & $11: 31-12: 15$ & 34.2 & 0.54 & 0.88 & 85 & 349.8 & 28 & $17.3-1284$ & $0.62 \times 0.41$ & 0.57 \\
\hline C/2012 S1 & 2 & 2013 Nov 17 & $12: 30-13: 27$ & 45.2 & 0.54 & 0.88 & 85 & 357.2 & 28 & $17.3-1284$ & $0.54 \times 0.40$ & 0.52 \\
\hline
\end{tabular}

Notes.

a On-source observing time.

b Heliocentric distance.

${ }^{c}$ Geocentric distance of the comet (JPL Horizons).

${ }^{\mathrm{d}}$ Sun-comet-observer (illumination phase) angle.

e Mean observational frequency.

${ }^{\mathrm{f}}$ Number of $(12 \mathrm{~m})$ antennae in the telescope array.

$\mathrm{g}$ Range of antenna baseline lengths.

h Angular resolution (dimensions of Gaussian fit to PSF) at $\bar{v}$, excluding antennae DV07, DV19, DV24, and DV25.

${ }^{\mathrm{i}}$ Median precipitable water vapor column length at zenith.

determination of its chemical origin is important for testing the role of comets in delivering prebiotic compounds to the early Earth (Oro \& Cosmovici 1997; DiSanti et al. 2006).

The most accurate method for determining the production site of a given cometary species is through measurement of its spatial distribution about the nucleus, especially its variation with nucleocentric distance in the innermost coma (within a few thousand kilometers of the nucleus). In this study, we report results from the first cometary observations using the Atacama Large Millimeter/Submillimeter Array (ALMA), and present spatially resolved measurements of the distributions of $\mathrm{HNC}$, $\mathrm{HCN}$, and $\mathrm{H}_{2} \mathrm{CO}$ within the comae of two comets originally from the Oort Cloud reservoir: C/2012 F6 (Lemmon) and C/2012 S1 (ISON).

\section{OBSERVATIONS}

Comet C/2012 F6 (Lemmon) is a long-period comet (orbital period approximately $11,000 \mathrm{yr}$; semi-major axis $a=493 \mathrm{AU}$ ) that reached perihelion on 2013 March 24. Comet C/2012 S1 (ISON) was a sungrazing comet, with $a \gtrsim 10,000 \mathrm{AU}$, and its orbit passed within $0.013 \mathrm{AU}$ of the Sun at perihelion on 2013 November 28.

Observations were made in Cycle 1 Early Science mode using the ALMA Band 7 receiver, covering frequencies between 338.6 and $364.6 \mathrm{GHz}(0.82-0.89 \mathrm{~mm})$. Comet Lemmon was observed 2013 June 1-2 and ISON was observed 2013 November 17 (Table 1). The cometary positions were tracked using JPL Horizons ephemerides (JPL\#45 for ISON and JPL\#22 for Lemmon). Two correlator settings permitted the simultaneous observation of two sets of spectral lines (plus continuum) for each comet (see Table 2). Weather conditions were excellent for all observations, with high atmospheric phase stability, and extremely low precipitable water vapor (zenith PWV = $0.44-0.83 \mathrm{~mm}$ ). Quasar observations were used for bandpass and phase calibration. Ceres and Titan were used to calibrate ISON's flux scale, and Pallas was used for Lemmon. The absolute flux calibration error is expected to be less than $15 \%$. The spatial resolution was 0.'4-0.9 (Table 1) and the channel spacing was $244 \mathrm{kHz}$, leading to a (Hanning smoothed) spectral resolution of about $0.42 \mathrm{~km} \mathrm{~s}^{-1}$.

The data were flagged, calibrated, and imaged using standard routines in CASA version 4.1.0 (McMullin et al. 2007). No signal was detected for baselines $\gtrsim 400 \mathrm{~m}$, so the most distant
Table 2

Detected Spectral Lines, Fluxes, Production Rates, and Parent Scale Lengths

\begin{tabular}{|c|c|c|c|c|c|c|}
\hline Species & Transition & $\begin{array}{c}\text { Frequency } \\
\quad(\mathrm{GHz})\end{array}$ & $\begin{array}{c}E_{u} \\
(\mathrm{~K})\end{array}$ & $\begin{array}{c}\text { Flux }^{\mathrm{a}} \\
\left(\mathrm{Jy} \mathrm{km} \mathrm{s}^{-1}\right)\end{array}$ & $\begin{array}{c}Q^{\mathrm{b}} \\
\left(10^{26} \mathrm{~s}^{-1}\right)\end{array}$ & $\begin{array}{c}L_{p}{ }^{\mathrm{c}} \\
(\mathrm{km})\end{array}$ \\
\hline \multicolumn{7}{|c|}{ C/2012 F6 (Lemmon) Setting 1} \\
\hline $\mathrm{H}_{2} \mathrm{CO}$ & $5_{1,5}-4_{1,4}$ & 351.769 & 62.5 & $0.86(8)$ & 2.1 & $1200_{-400}^{+1200}$ \\
\hline $\mathrm{HCN}$ & $4-3$ & 354.505 & 42.5 & $8.55(9)$ & 2.3 & $<50$ \\
\hline \multicolumn{7}{|c|}{ C/2012 F6 (Lemmon) Setting 2} \\
\hline $\mathrm{HNC}$ & $4-3$ & 362.630 & 43.5 & $0.38(9)$ & $\sim 0.1$ & $\ldots^{d}$ \\
\hline \multicolumn{7}{|c|}{ C/2012 S1 (ISON) Setting 1} \\
\hline $\mathrm{HCN}$ & $4-3$ & 354.505 & 42.5 & $11.76(9)$ & 3.5 & $<50$ \\
\hline \multicolumn{7}{|c|}{ C/2012 S1 (ISON) Setting 2} \\
\hline $\mathrm{H}_{2} \mathrm{CO}$ & $5_{1,5}-4_{1,4}$ & 351.769 & 62.5 & $3.79(10)$ & 16.4 & $280_{-50}^{+50}$ \\
\hline $\mathrm{HNC}$ & 4-3 & 362.630 & 43.5 & $1.92(13)$ & 1.2 & $700_{-400}^{+1100}$ \\
\hline
\end{tabular}

Notes.

a Integrated line flux within a 5 " diameter circular aperture centered on the comet; $1 \sigma$ errors on trailing digits given in parentheses.

${ }^{\mathrm{b}}$ Best-fit production rate from visibility model (errors are $\sim \pm 10 \%$ ).

${ }^{c}$ Best-fit parent scale length, including $\pm 1 \sigma$ errors.

${ }^{\mathrm{d}}$ Insufficient signal-to-noise for fit.

antennae (DV07, DV19, DV24, and DV25; >500 $\mathrm{m}$ from the array center) were excluded during imaging. Deconvolution of the point-spread function (PSF) was performed using the Högbom algorithm, with natural visibility weighting and a flux threshold of twice the rms noise in each image. Finally, the deconvolved images were convolved with a Gaussian fit to the PSF. The continuum peak of comet Lemmon was offset by a (negligible) 0.'9 NE of image center, whereas ISON was offset 6.'5 NW (explainable as a result of non-gravitational acceleration; Sekanina \& Kracht 2014). Images of ISON were therefore corrected for the response of the ALMA primary beam (half-power beam-width $\approx 17^{\prime \prime} .5$ ).

Images were transformed from celestial coordinates to cometocentric (projected) spatial distances, the origin of which was determined as the location of peak continuum flux.

\section{MOLECULAR MAPS AND RADIAL PROFILES}

The detected spectral lines, including upper-state energies $\left(E_{u}\right)$ and integrated fluxes, are summarized in Table 2. Figure 1 

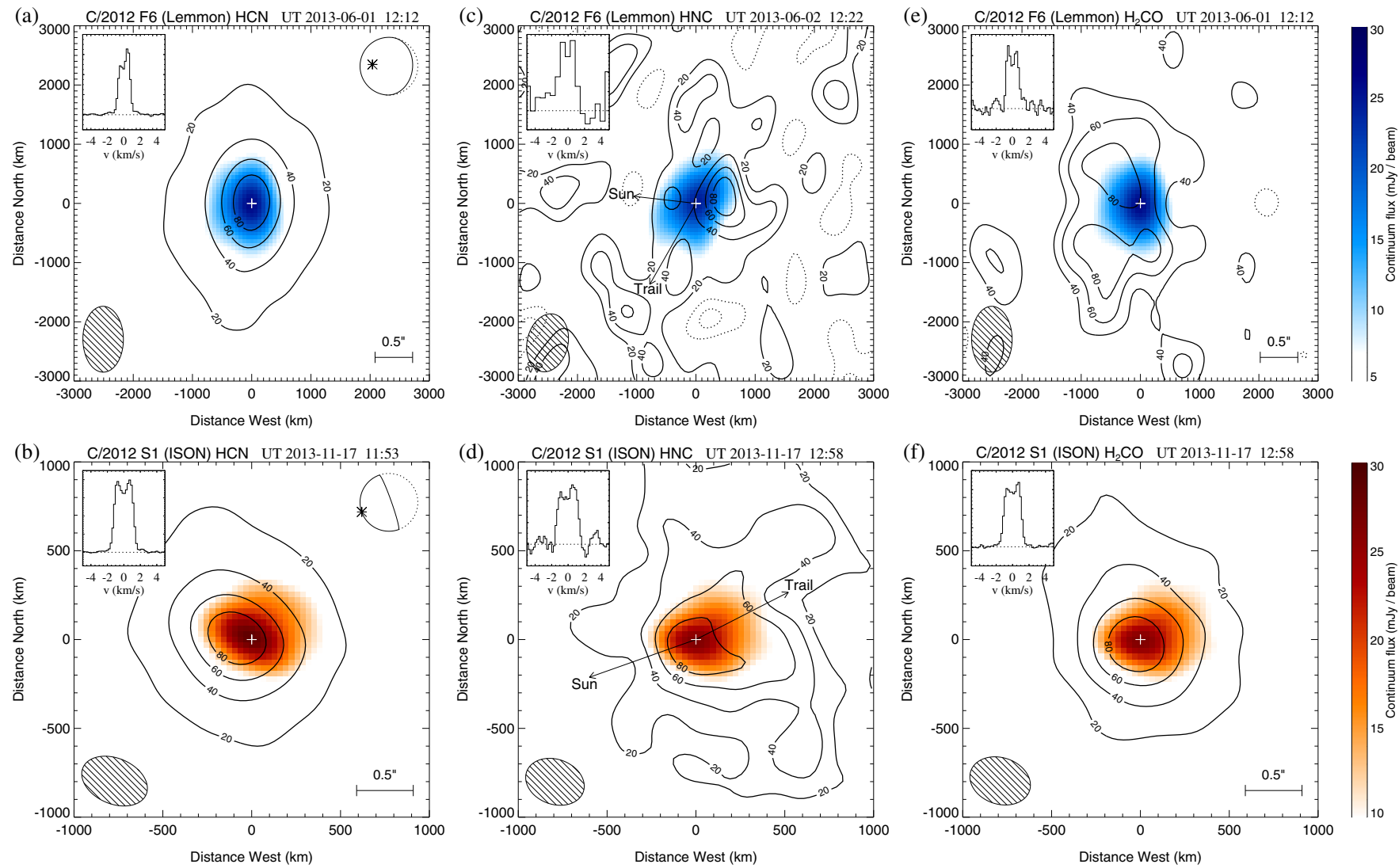

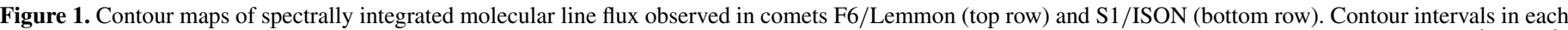

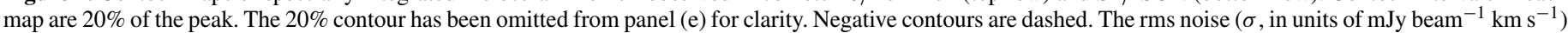

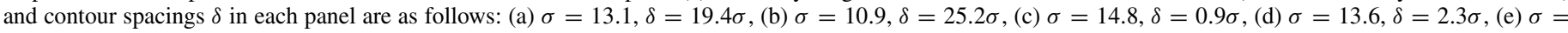

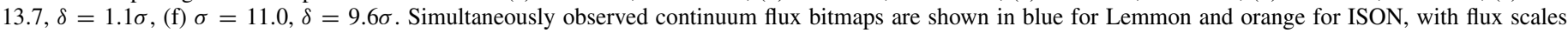

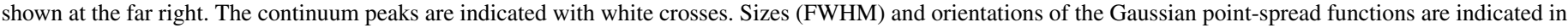

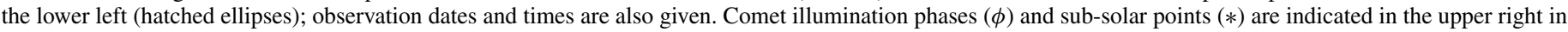

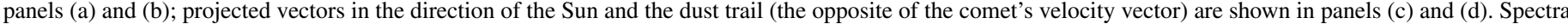

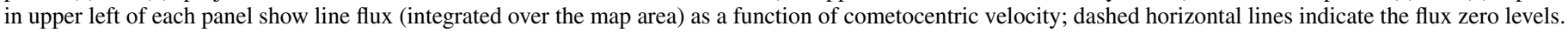

(A color version and supplemental data of this figure are available in the online journal.)

shows spectrally integrated flux contour maps for the observed molecules in each comet, overlaid on bitmap images of the (simultaneously observed) continuum emission.

Dramatic differences are evident between different molecular species observed in the same comet, and between the same species observed in the different comets. By eye, the HCN distributions in both comets appear quite rotationally symmetric about the central peak. For Lemmon, no offset between the $\mathrm{HCN}$ and continuum peaks is distinguishable, whereas ISON's HCN peak is offset $80 \mathrm{~km}$ eastward of the continuum peak. Both comets show a compact, strongly peaked submillimeter continuum, but ISON exhibits an additional, fainter, tail-like feature extending to the northwest, in approximately the opposite direction to the comet's motion (as marked by the "trail" vector in Figure 1(d)). Cometary submillimeter emission is likely due to large dust grains, $\gtrsim 1 \mathrm{~mm}$ in size (Jewitt \& Luu 1992), so ISON's submillimeter tail is consistent with the presence of a debris stream following behind the comet's orbit, as discussed by Sekanina \& Kracht (2014).

For HNC, the emission observed in comet Lemmon (detected at $4.4 \sigma$ confidence) is offset from the continuum peak by $500 \pm$ $150 \mathrm{~km}$ to the west (Figure 1(c)) - in an approximately antisunward direction. By contrast, ISON's HNC peak (Figure 1(d)) lies very close to (within $100 \mathrm{~km}$ of) the continuum peak. The HNC map for ISON shows a wealth of remarkable extended spatial structure, with at least three streams (identified at $>6 \sigma$ confidence), emanating away from the main peak. The majority of HNC emission from both comets is asymmetric, originating predominantly in the anti-sunward hemispheres of their comae.

Formaldehyde also shows strikingly different distributions for comets Lemmon and ISON (Figures 1(e) and (f)), highlighting the complex nature of this species. Lemmon has a remarkably flat and extended $\mathrm{H}_{2} \mathrm{CO}$ map, as demonstrated by the size of the region traced by the $40 \%$ contour compared with the other maps. The mean FWHM of the $\mathrm{H}_{2} \mathrm{CO}$ distribution is $\bar{d}=2920 \mathrm{~km}$ (which is 3.5 times the instrumental PSF value of $\bar{d}=840 \mathrm{~km}$ ), and is significantly broader than both HCN and the continuum, which have $\bar{d}=1480 \mathrm{~km}$ and $1180 \mathrm{~km}$, respectively. Lemmon's $\mathrm{H}_{2} \mathrm{CO}$ map shows two main emission peaks at distances $\sim 500-1000 \mathrm{~km}$ from the continuum peak (although noise is likely responsible for some of the structure in this map). By contrast, the $\mathrm{H}_{2} \mathrm{CO}$ distribution for comet ISON is dominated by a strong, compact central peak (with $\bar{d}=600 \mathrm{~km}$, compared to $\bar{d}=320 \mathrm{~km}$ for the PSF), and has a relatively symmetrical contour pattern, similar to $\mathrm{HCN}$.

Further insight into the flux distributions can be obtained by comparison of the azimuthally averaged radial flux profiles (Figure 2). For each map, the HCN flux peak was taken as the origin (except for the continuum maps, for which the continuum peak was used). The average fluxes inside successive 0 ' 05 thick 

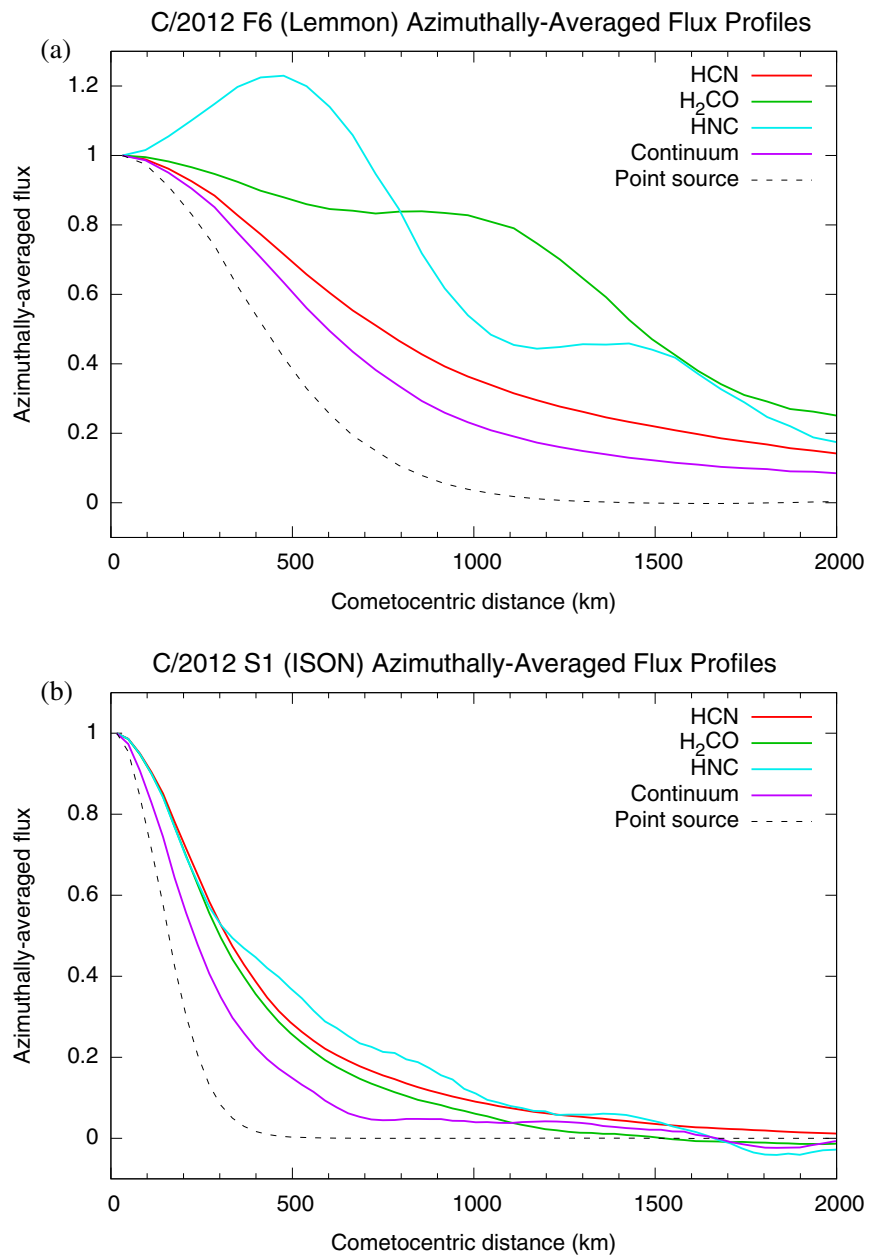

Figure 2. Azimuthally averaged flux profiles for (a) Lemmon and (b) ISON. Profiles have been normalized to unity at the origin. The $354 \mathrm{GHz}$ continuum profile for the (point-source) bandpass calibrators used for each comet are shown with dotted lines.

(A color version of this figure is available in the online journal.)

annuli are plotted as a function of annulus radius, normalized to unity in the first annulus. These azimuthally averaged profiles are dominated by the $1 / \rho$ decay that would be expected from uniform, isotropic expansion (where $\rho$ is the projected radial coordinate). Their shapes are also affected by flux losses due to a lack of short baselines in the array, which become progressively greater for larger structures, and hence, larger cometocentric distances. Despite significant noise ripples, the relatively broad $\mathrm{HNC}$ and $\mathrm{H}_{2} \mathrm{CO}$ profiles for Lemmon (and $\mathrm{HNC}$ for ISON) are indicative of coma production for these species. For ISON, the $\mathrm{H}_{2} \mathrm{CO}$ curve lies inside that of $\mathrm{HCN}$, which is a natural consequence of the shorter lifetime of $\mathrm{H}_{2} \mathrm{CO}$ (1280 s versus 23,100 s for $\mathrm{HCN}$ at $0.54 \mathrm{AU}$; Huebner et al. 1992). The cause of the relatively narrow continuum profile for both comets will be discussed in more detail in a future paper.

\section{LOCATIONS OF MOLECULAR PRODUCTION/RELEASE}

Following the method of Boissier et al. (2007, 2014), the interferometric visibility amplitudes for the observed molecules were modeled under the assumption of uniform, isotropic outflow. In this (Haser 1957) paradigm, each molecular distribution is determined by the production rate $(Q)$, outflow velocity $(v)$, parent scale length $\left(L_{p}\right)$, and (for daughter species) the photodissociation rate, with $Q$ and $L_{p}$ as free parameters. Respective outflow velocities of $1.0 \mathrm{~km} \mathrm{~s}^{-1}$ and $0.7 \mathrm{~km} \mathrm{~s}^{-1}$ for ISON and Lemmon were obtained from the half-width at half-maximum of the HCN lines. For ISON, a gas kinetic temperature $T=90 \mathrm{~K}$ was adopted (Agúndez et al. 2014). For Lemmon, $T=55 \mathrm{~K}$ was obtained by scaling the measurement of Biver et al. (2014) assuming $T \propto r_{\mathrm{H}}^{-1}$. The molecular excitation calculation considers collisions with $\mathrm{H}_{2} \mathrm{O}$ and electrons, and pumping by solar infrared radiation. Goodness of fit was determined by minimizing the sum of the squares of the differences between the real parts of the observed and modeled visibility amplitudes. The best-fitting models for the observed visibilities are shown in Figure 3, and corresponding $Q$ and $L_{p}$ values are given in Table 2.

\subsection{HCN and HNC}

The presented maps and visibilities provide clear evidence regarding the origins of the observed molecules. The comparison between $\mathrm{HCN}$ and $\mathrm{HNC}$ is particularly revealing. As shown in Figure 3, our visibility data are most consistent with the production of $\mathrm{HCN}$ as a primary species, released from (or very near to) the nuclei of both comets. By contrast, for HNC in comet ISON a distributed source is required (with $L_{p}=300-1800$ at $\left.r_{\mathrm{H}}=0.54 \mathrm{AU}\right)$. The HNC visibilities in the comet Lemmon are also consistent with a distributed source, although the error bars are large, so a parent model fits this data equally well.

Figure 1(d) shows significant quantities of HNC in streams/ clumps at projected distances up to $1000 \mathrm{~km}$ from the nucleus of comet ISON that are not present in HCN. These suggest that HNC is released in collimated/anisotropic outflows. The presence of offset HNC emission in Lemmon's coma is also consistent with this hypothesis. From interferometric observations of comet C/1995 O1 (Hale-Bopp), Wink et al. (1997) found evidence for coma production of HNC, and Blake et al. (1999) identified $\mathrm{HNC}$ release in jets, whereas $\mathrm{HCN}$ was released from (near to) the nucleus. Variations in excitation cannot plausibly explain the observed differences between these molecules because of the near-identical upper-state energies of the observed HCN and HNC transitions (Table 2).

Our measured $\mathrm{HCN}$ production rate of $2.3 \times 10^{26} \mathrm{~s}^{-1}$ in comet Lemmon (at $r_{\mathrm{H}}=1.47 \mathrm{AU}$ on 2013 June 1) is in agreement with that observed by Paganini et al. (2014) (at $r_{\mathrm{H}}=1.74 \mathrm{AU}$ on 2013 June 20). For ISON, our value of $3.5 \times 10^{26} \mathrm{~s}^{-1}$ on 2013 November 17 is consistent with the value of $3.6 \times 10^{26} \mathrm{~s}^{-1}$ measured by Agúndez et al. (2014) two days earlier. Our $Q(\mathrm{HNC}) / Q(\mathrm{HCN})$ ratio of $34 \%$ is somewhat larger than the value of $18 \%$ found by Agúndez et al. (2014), which may be indicative of variations in the relative $\mathrm{HCN}$ and $\mathrm{HNC}$ production rates with time. The $Q(\mathrm{HNC}) / Q(\mathrm{HCN})$ ratio in comet Lemmon was only about $0.4 \%$, which is a factor of $\sim 85$ less than in comet ISON. In a sample of 14 moderately active comets, Lis et al. (2008) found a similarly strong change in this ratio with heliocentric distance. The strong dependence of HNC production rate on $r_{\mathrm{H}}$ (see also Irvine et al. 1998), and its asymmetric spatial distribution, imply release of HNC from a refractory component of the nucleus, ejected into the coma in anisotropic streams.

\section{2. $\mathrm{H}_{2} \mathrm{CO}$}

Formaldehyde $\left(\mathrm{H}_{2} \mathrm{CO}\right)$ has a clear distributed source in both comets. Visibility modeling reveals a parent scale length of 

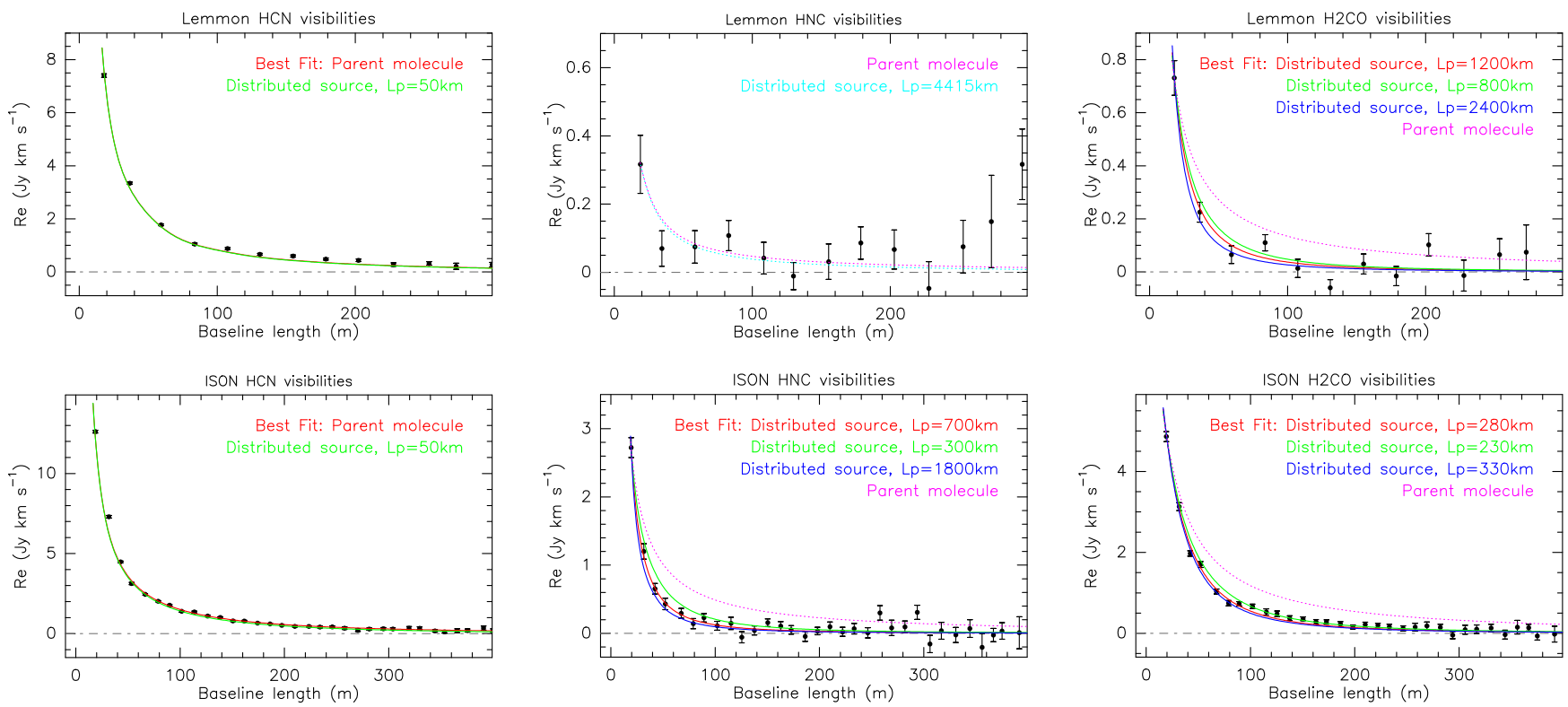

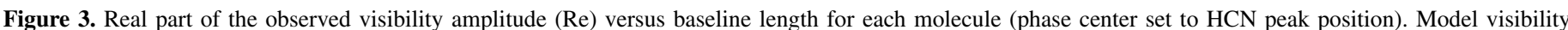

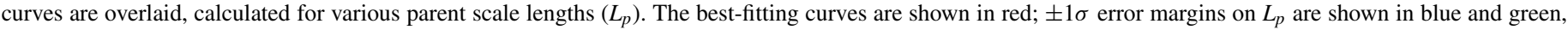
respectively; purple dotted indicates best-fitting parent $\left(L_{p}=0\right)$ curves in those cases for which a distributed source $\left(L_{p} \neq 0\right)$ fits best.

(A color version of this figure is available in the online journal.)

$L_{p}=800-2400 \mathrm{~km}$ for Lemmon and $L_{p}=230-330 \mathrm{~km}$ for ISON (corresponding to photodissociation rates of $\Gamma \approx$ $6 \times 10^{-4} \mathrm{~s}^{-1}$ and $\approx 4 \times 10^{-3} \mathrm{~s}^{-1}$, respectively). Considering the eight-fold increase in solar insolation expected at ISON's heliocentric distance ( $0.54 \mathrm{AU})$ compared with Lemmon's (1.47 AU), the order-of-magnitude difference in $\Gamma$ is consistent with optically thin photodissociation of the parent in a uniform outflow. This suggests that the parent of $\mathrm{H}_{2} \mathrm{CO}$ could be an (unknown) organic molecule or simple-addition polymer of low number (Cottin \& Fray 2008).

Several previous studies have identified $\mathrm{H}_{2} \mathrm{CO}$ release in the coma (e.g., Biver et al. 1999; Cottin et al. 2004), and our result is qualitatively similar to those. The parent scale lengths we derived are smaller than previous estimates, which were in the range $(4000-8000) r_{\mathrm{H}}^{1.5} \mathrm{~km}$. The results may, however, be consistent given that our observations probe only the inner few thousand kilometers of the coma, whereas previous studies probed distances $\gtrsim 2000 \mathrm{~km}$. Due to the lack of short baselines, our ALMA data cannot rule out the presence of additional $\mathrm{H}_{2} \mathrm{CO}$ sources with angular sizes $\gtrsim 5^{\prime \prime}$. We also note that for previous radio measurements, observational and model uncertainties (particularly the $\mathrm{H}_{2} \mathrm{CO}$ excitation), were significant; the latter are less important for our observations, which probe the densest part of the coma where departures from local thermodynamic equilibrium are minimized.

The $\mathrm{H}_{2} \mathrm{CO}$ production rates we derived for Lemmon (Table 2) are compatible with the upper limit of $2.5 \times 10^{26} \mathrm{~s}^{-1}$ from Paganini et al. (2014) on June 20. ISON's $\mathrm{H}_{2} \mathrm{CO}$ production rate was 6.9 times greater, despite only a factor of 1.5 increase in HCN. Similar to HNC, previous observations (Fray et al. 2006) identified a stronger $r_{\mathrm{H}}$ dependence for $Q\left(\mathrm{H}_{2} \mathrm{CO}\right)$ than for $Q(\mathrm{HCN})$, which is consistent with our results, although the difference in $Q\left(\mathrm{H}_{2} \mathrm{CO}\right)$ between Lemmon and ISON could also be attributed (at least in part) to a difference in the chemical composition of the nucleus.

By considering the thermal degradation properties of Polyoxymethylene (POM) embedded in organic grains, Cottin et al. (2004) and Fray et al. (2006) successfully modeled the observed
$\mathrm{H}_{2} \mathrm{CO}$ parent scale length in $1 \mathrm{P} /$ Halley and the $\mathrm{H}_{2} \mathrm{CO}$ production rate as a function of $r_{\mathrm{H}}$ in Hale-Bopp, respectively. The scale length of POM depends strongly on the sizes and temperatures of the grains. Using the model of Fray et al. (2006) assuming a grain outflow velocity and temperature that vary as $r_{\mathrm{H}}^{-0.5}$, for Lemmon, our observed $\mathrm{H}_{2} \mathrm{CO}$ scale length is consistent with grains a few microns in diameter, whereas for ISON, grain sizes $\gtrsim 40 \mu \mathrm{m}$ are required. In reality, an ensemble of grain sizes, temperatures, and velocities will be present, necessitating detailed modeling to confirm if thermal degradation of POM matches our $\mathrm{H}_{2} \mathrm{CO}$ observations.

\section{CONCLUSION}

We have presented ALMA measurements of molecular line and continuum emission from two Oort Cloud comets: C/2012 F6 (Lemmon) and C/2012 S1 (ISON). These data reveal the detailed spatial structures and origins of $\mathrm{HCN}, \mathrm{HNC}, \mathrm{H}_{2} \mathrm{CO}$, and dust within the innermost few thousand kilometers of the cometary comae. For both comets, the dominant source of $\mathrm{HCN}$ was from (or very close to) the nucleus. By contrast, the HNC distributions suggest production from a source entering the coma in anisotropic, clumpy stream(s). A distributed $\mathrm{H}_{2} \mathrm{CO}$ source was identified in both comets. The scale length of the putative $\mathrm{H}_{2} \mathrm{CO}$ parent was on the order of a few hundred kilometers for comet ISON and a few thousand kilometers for Lemmon, consistent with a parent destruction rate that scales with the intensity of solar radiation. Relative to $\mathrm{HCN}$, comet ISON's coma was about an order of magnitude richer in $\mathrm{H}_{2} \mathrm{CO}$ and HNC than that of comet Lemmon, consistent with a more rapid production of these molecules at ISON's smaller heliocentric distance.

The release of $\mathrm{HNC}$ and $\mathrm{H}_{2} \mathrm{CO}$ as product species implies the existence of chemical precursor materials in the coma, which undergo sublimation, photochemical, and/or thermal degradation to produce the observed molecules in the gas phase. Heating or photolysis of refractory materials such as dust grains, polymers, or other macro-molecules, and their subsequent 
breakdown at distances $\sim 100-10,000 \mathrm{~km}$ from the nucleus presents the most compelling hypothesis for the origin of the observed $\mathrm{H}_{2} \mathrm{CO}$ and $\mathrm{HNC}$. The presence (and composition) of the hypothesized macro-molecular precursors will be measured by the COSIMA instrument on the Rosetta spacecraft during its encounter with comet 67P/Churyumov-Gerasimenko in 2014 (Kissel et al. 2007; Le Roy et al. 2012).

The interferometric data presented in this Letter show that routine high-resolution observations of the distributions of molecules and dust grains in cometary comae are now possible. These observations pave the way for future measurements of spatially, spectrally, and temporally resolved coma emission, from which presently little understood properties such as the detailed physical structure of the coma (on size scales of several hundred kilometers), and the molecular release and reaction mechanisms, will be derived.

This work was supported by the NASA Astrobiology Institute through the Goddard Center for Astrobiology, and NASA's Planetary Atmospheres and Planetary Astronomy Programs. It makes use of the following ALMA data: ADS/JAO.ALMA \#2012.A.00020.S and \#2012.A.00033.S. ALMA is a partnership of ESO (representing its member states), NSF (USA), and NINS (Japan), together with NRC (Canada) and NSC and ASIAA (Taiwan), in cooperation with the Republic of Chile. The Joint ALMA Observatory is operated by ESO, AUI/NRAO, and NAOJ. The National Radio Astronomy Observatory is a facility of the National Science Foundation operated under cooperative agreement by Associated Universities, Inc. D.C.L. is supported by NASA through JPL/Caltech. D.M. is supported by Basal CATA PFB-06 and the ICM MAS. Y.J.K. is supported by NSC grants 99-2112-M-003-003-MY3 and 100-2119-M-003001-MY3.

\section{REFERENCES}

Agúndez, M., Biver, N., Santos-Sanz, P., Bockelée-Morvan, D., \& Moreno, R. 2014, A\&A, 564, L2

Blake, G. A., Qi, C., Hogerheijde, M. R., Gurwell, M. A., \& Muhleman, D. O. 1999, Natur, 398, 213

Boissier, J., Bockeleé-Morvan, D., Biver, N., et al. 2007, A\&A, 475, 1131

Boissier, J., Bockelée-Morvan, D., Biver, N., et al. 2014, Icar, 228, 197

Biver, N., Bockelée-Morvan, D., Colom, P., et al. 1997, Sci, 275, 1915

Biver, N., Bockelée-Morvan, D., Crovisier, J., et al. 1999, AJ, 118, 1850

Biver, N., Bockelée-Morvan, D., Debout, V., et al. 2014, A\&A, 566, L5

Biver, N., Debout, V., Bockelée-Morvan, D., et al. 2013, AAS, 45, 502.03 (DPS Meeting)

Cottin, H., Bénilan, Y., Gazeau, M.-C., \& Raulin, F. 2004, Icar, 167, 397

Cottin, H., \& Fray, N. 2008, SSRv, 138, 179

DiSanti, M. A., Bonev, B. P., Magee-Sauer, K., et al. 2006, ApJ, 650, 470

Ehrenfreund, P., \& Charnley, S. B. 2000, ARA\&A, 38, 427

Fray, N., Bénilan, Y., Biver, N., et al. 2006, Icar, 184, 239

Haser, L. 1957, BSRSL, 43, 740

Huebner, W. F., Keady, J. J., \& Lyon, S. P. 1992, Ap\&SS, 195, 1

Irvine, W. M., Bergin, E. A., Dickens, J. E., et al. 1998, Natur, 393, 547

Irvine, W. M., Bockeleé-Morvan, D., Lis, D. C., et al. 1996, Natur, 383, 418

Jewitt, D., \& Luu, J. 1992, Icar, 100, 187

Kissel, J., Altwegg, K., Clark, B. C., et al. 2007, SSRv, 128, 823

Le Roy, L., Briani, G., \& Briois, C. 2012, P\&SS, 65, 83

Lis, D. C., Bockeleé-Morvan, D., Boissier, J., et al. 2008, ApJ, 675, 931

McMullin, J. P., Waters, B., Schiebel, D., Young, W., \& Golap, K. 2007, in ASP Conf. Ser. 376, Astronomical Data Analysis Software and Systems XVI, ed. R. A. Shaw, F. Hill, \& D. J. Bell (San Francisco, CA: ASP), 127

Milam, S. N., Remijan, A. J., Womack, M., et al. 2006, ApJ, 649, 1169

Mumma, M. J., \& Charnley, S. B. 2011, ARA\&A, 49, 471

Oro, J., \& Cosmovici, C. B. 1997, in Proceedings of the 5th International Conference on Bioastronomy, Astronomical and Biochemical Origins and the Search for Life in the Universe, ed. C. B. Cosmovici, S. Bowyer, \& D. Werthimer (Bologna, Italy: Editrice Compositori), 97

Paganini, L., Disanti, M. A., Mumma, M. J., et al. 2014, AJ, 147, 15

Rodgers, S. D., \& Charnley, S. B. 2001, MNRAS, 323, 84

Rodgers, S. D., \& Charnley, S. B. 2005, MNRAS, 356, 1542

Sekanina, Z., \& Kracht, R. 2014, ApJ, submitted

Wink, J., Bockelée-Morvan, D., Despois, D., et al. 1997, EM\&P, 78, 63 